\title{
Unveiling Uncertain Forces in Second-order Driven Oscillators Via Internal Model: A Discrete-time feedback
}

\author{
R. FEMAT* and C. JIMÉNEZ-GALLEGOS \\ Universidad Autónoma de San Luis Potosí Apdo Postal 3-90, 78231 San Luis Potosí, S.L.P., México
}

(Received 17 February 2000)

\begin{abstract}
Unveil uncertain forces acting into (or onto) systems is a very interesting and old problem. Indeed, a lot effort has been devoted to develop procedures which results in the understanding of the uncertain forces and its effects. This contribution deals with recovering of the dynamics of the uncertain forces from measurements (time series). The main idea is to construct an internal model of the nonlinear system and design a discretetime feedback in such way that the model/system differences be stabilized at origin. In principle, if the internal model tracks the trajectory of the nonlinear system, then the uncertain force is recovered by the stabilizing command.
\end{abstract}

Keywords: Discrete-time feedback; Dynamic uncertainties estimation

\section{INTRODUCTION}

Unveiling the forces acting into (or onto) systems is not an easy task. In fact, a lot effort has been devoted to unveil the hidden secrets of nature. The mathematical model development searches knowledge in this direction. Several theories have been proposed. There are some alternatives from the probability theory (for instance, Synergetics [1]) whereas another one departs from the deterministic and operators theory (see, for example, [2] and [3]). However, mathematical models have some limitations; hence estimation procedures are desired.
Recently, estimation procedures have been taken from control theory. For instance, $a b$ servability property [4] of the nonlinear systems can be exploited to get estimated values of unmeasured states. The observability property strongly depends on the choice of the measured state (which is so-called system output if measurements are continuous [4] and time series if measurements are discrete-time [5,6]). The main idea behind observability is the phase-space reconstruction; i.e., the history of all states is into the time series [7]. The main application of the observability property has been to reconstruct, if exists, the attractor of the dynamical

*Corresponding author. Tel.: (+52-4) 826-2440 ext. 105, e-mail: rfemat@deimos.tc.uaslp.mx 
system [5,6]. However, in [4] was discussed that observability property can be exploited to perform chaos synchronization. This is, if the history of all states is into the time series and the observability property conditions are satisfied, then the hidden states can be dynamically reconstructed. For example, according to Takens theorem [7], it is possible to reconstruct an embedding of a time series in a phase-space in absence of noisy measurements [5] by using timedelay coordinates. In addition, the time delay coordinates can be employed as estimated values of a dynamical system to yield chaos suppression [8]. Nevertheless, such state reconstruction has some constraints, which can be fundamentally seen as sensitivity tradeoffs [9].

On the other hand, from the practical point of view, there is an important problem. Can the uncertain forces be reconstructed from the measured state?. In principle, since the history of the system is in the time series and the dynamical series are yielded by forces acting into (or onto) the system, hence the unknown forces can be unveiled from the time series. Indeed, unveil uncertain forces is an interesting and old problem for physicist. Some a priori information is often required; for example, the order of the system, smoothness or boundedness. Besides, some information regarding noise or disturbances can be useful. Nevertheless, the more interesting case is to unveil uncertain forces against the least prior knowledge about the system.

A force unveiling procedure is presented in this contribution. The main idea is to construct a dynamic feedback, which comprises a control law and a discrete-time estimator. The estimator and control cannot be separately designed. This is, the coupling between the control law and the discrete-time estimator yields the unveiling of the unknown force. The state estimator is based on the time-delays coordinates from time series whereas the control law structure is a stabilizing command.

\section{PROBLEM STATEMENT}

Consider the following nonlinear dynamical system

$$
\begin{gathered}
\dot{x}_{1, M}=x_{2, M} \\
\dot{x}_{2, M}=F_{M}\left(x_{M} ; p_{M}\right)+T_{M}\left(t ; r_{M}\right)+\alpha(x(t)) \\
y_{M}=C x_{M}
\end{gathered}
$$

where $x_{M} \in \mathbb{R}^{2}$ is a state vector, $F_{M}\left(x_{M} ; p_{M}\right)$ is a smooth function (which could be uncertain), $T_{M}$ $\left(t ; r_{M}\right)$ is an external perturbation term (which could be also uncertain) and $p_{M}, r_{M}$ are parameter set and $y_{M} \in \mathbb{R}$ is the system output (time series). Without lost of generality, one can denote $\alpha(x(t))$ as the unknown force.

Note that uncertain forces can be acting into (or onto) the system. For example, let us assume that $\alpha(x) \equiv 0$ and $F_{M}\left(x_{M} ; p_{M}\right)$ or $T_{M}\left(t ; r_{M}\right)$ is uncertain, the force to be unveiled is acting into the system. In same way, if $F_{M}\left(x_{M} ; p_{M}\right)$ and $T_{M}\left(t ; r_{M}\right)$ were known functions, hence $\alpha(x) \not \equiv 0$ represents the uncertain force, which should be unveiled.

Let us now take an almost-exact copy of the system (1.a), which is so-called mathematical model

$$
\begin{gathered}
\dot{x}_{1, S}=x_{2} \\
\dot{x}_{2, S}=F_{S}\left(x_{S} ; p_{S}\right)+T_{S}\left(t ; r_{S}\right)+u \\
y_{S}=C x_{S}
\end{gathered}
$$

where $u \in \mathbb{R}$ is an external force (control law). $F_{s}\left(x_{s} ; p_{s}\right)$ and $T_{s}\left(t ; p_{s}\right)$ are known and bounded functions; however; $p_{M} \neq p_{s}$ and $r_{M} \neq r_{M}$.

The unveiling problem is: given the system output $y_{M}$ and least prior knowledge of the structure of the nonlinear system (1.a), design an feedback scheme such that the uncertain forces can be unveiled. In some sense, the unveiling problem seems as a synchronization one where the actual system (1.b) can be seen as the so-called "master" whereas the mathematical model (1.c) represents the "slave" system. In others words, 
the synchronization objective is that $y_{s}$ tracks $y_{M}$. which implies that $x(t) \rightarrow 0 \Rightarrow x_{S}(t) \rightarrow x_{M}(t)$. This implies that $F_{s}\left(x_{s}, t ; p_{s}\right) \rightarrow F_{M}\left(x_{M}, t ; p_{M}\right)$ and $T_{s}\left(t ; r_{s}\right) \rightarrow T_{s}\left(t ; r_{s}\right)$ therefore $\alpha(x(t))-u \rightarrow 0$. From the control theory viewpoint, the synchronization problem can be interpreted as follows. Let us define $x \in \mathbb{R}^{n}$ such that $x_{i}=x_{i, M}-x_{i, s}$ for $i=$ $1,2,3, \ldots, n$. In this way, the following dynamical system describes the dynamics of the model error

$$
\begin{aligned}
\dot{x}_{1} & =x_{2} \\
\dot{x}_{2} & =\Delta F\left(x, t ; p_{1}\right)+\Delta T\left(t ; p_{2}\right)+\alpha(x)-u \\
y & =C x
\end{aligned}
$$

where $\Delta F\left(x ; p_{1}\right)=F_{M}\left(x_{M} ; p_{M}\right)-F_{s} \quad\left(x_{s} ; p_{s}\right) \quad$ and $\Delta T\left(t ; p_{2}\right)=T_{M}\left(t ; r_{M}\right)-T_{s}\left(t, r_{s}\right) \quad$ are uncertain smooth functions, which represent the mismatches between dynamical system and its model. Now, since the unveiling problem can be seen as a synchronization one and, from the control theory point of view, the synchronization problem can be understood as the stabilization of the system (2) at the origin. Hence, the goal is to find a feedback control law $u=u(x, t)$ such that $\lim x \rightarrow 0$ as $t \rightarrow \infty$.

\section{THE PROPOSED UNVEILING SCHEME}

\subsection{Dynamic Evolution in an Extended Space}

Following the ideas reported in [10], let us define $\eta=\Delta F\left(x ; p_{1}\right)+\Delta T\left(t ; p_{2}\right)+\alpha(x)$. Then the model error system can be rewritten as follows

$$
\begin{gathered}
\dot{x}_{1}=x_{2} \\
\dot{x}_{2}=\eta+u \\
\dot{\eta}=\Gamma(x, \eta, u, t ; \pi)
\end{gathered}
$$

where $\Gamma(x, \eta, u, t ; \pi)=x_{2} \partial_{1}\left(\Delta F\left(x ; p_{1}\right)+\Delta T\left(t ; p_{2}\right)+\right.$ $\alpha(x))+[\eta+u] \partial_{2}\left(\Delta F\left(x ; p_{1}\right)+\Delta T\left(t ; p_{2}\right)+\alpha(x)\right)+$ $\dot{u}, \pi$ is the parameters set.
It has been proved that, under feedback output control, the a second-order driven oscillator can be stabilized at origin (see Appendix in [10]). In addition, has been proved that the system (3) is externally dynamically equivalent to the system (2). This is, there is a time-invariant manifold, $\Psi(x$, $\eta, t ; \pi)$, such that the solution of the system (2) is a projection of the system (3) as long as the initial conditions be $\Psi(x(0), \eta(0), 0 ; \pi) \equiv 0$, which is satisfied by definition (see Appendix in [10]). In order to illustrate the geometrical interpretation of the augmented state, $\eta$, we have selected the Duffing equation. To perform the numerical simulations, we have defined the augmented state as follows: $\eta \equiv-\delta x_{2}+x_{1}-x_{1}^{3}+A \cos (\omega t)$. In this way, one has that $\Gamma\left(x_{1}, x_{2}, \eta, u, t\right)=-\delta(\eta+u)+x_{2}-3 x_{1}^{2} x_{2}-$ $A \omega \sin (\omega t)$. Figure 1 shows the phase portrait of the systems (2) and (3). The initial conditions were chosen as follows: for system (2) $x(0)=(0.0,1.0)$ while for system (3) $\left(x_{1}(0), x_{2}(0), \eta(0)\right)=(0.0$, 1.0.0.01). The same parameters values were chosen for both systems.

Since systems (2) and (3) are dynamically equivalent, hence a feedback can be designed from the system (3) in such way that trajectories of the system (2) be leaded to origin. In fact, Eq. (3) is used as an intermediate system toward the construction of the feedback. The main idea is the following. If one is able to stabilize the trajectories of the system (3) neither measurements of the velocity, $x_{2}$, nor the augmented state, $\eta$, then the trajectories of the system (2) will be leaded to origin (prescribed point) against the uncertain terms.

\subsection{The Discrete-time Feedback Via Uncertainties Estimation}

Now, let us consider the ideal feedback, which can be obtained from system (2) as follows: $u=-\eta+k_{2} x_{2}+k_{1} x_{1}$, where, by definition, $\eta=$ $\Delta F\left(x ; p_{1}\right)+\Delta T\left(t ; p_{2}\right)+\alpha(x)$. Under such ideal feedback the system (2) becomes stable at origin if polynomial $P_{2}(s)=s^{2}+k_{2} s+k_{1}$ has its roots at lefthand complex plane. This implies that the system (3) is also stable at origin. Nevertheless, the ideal 


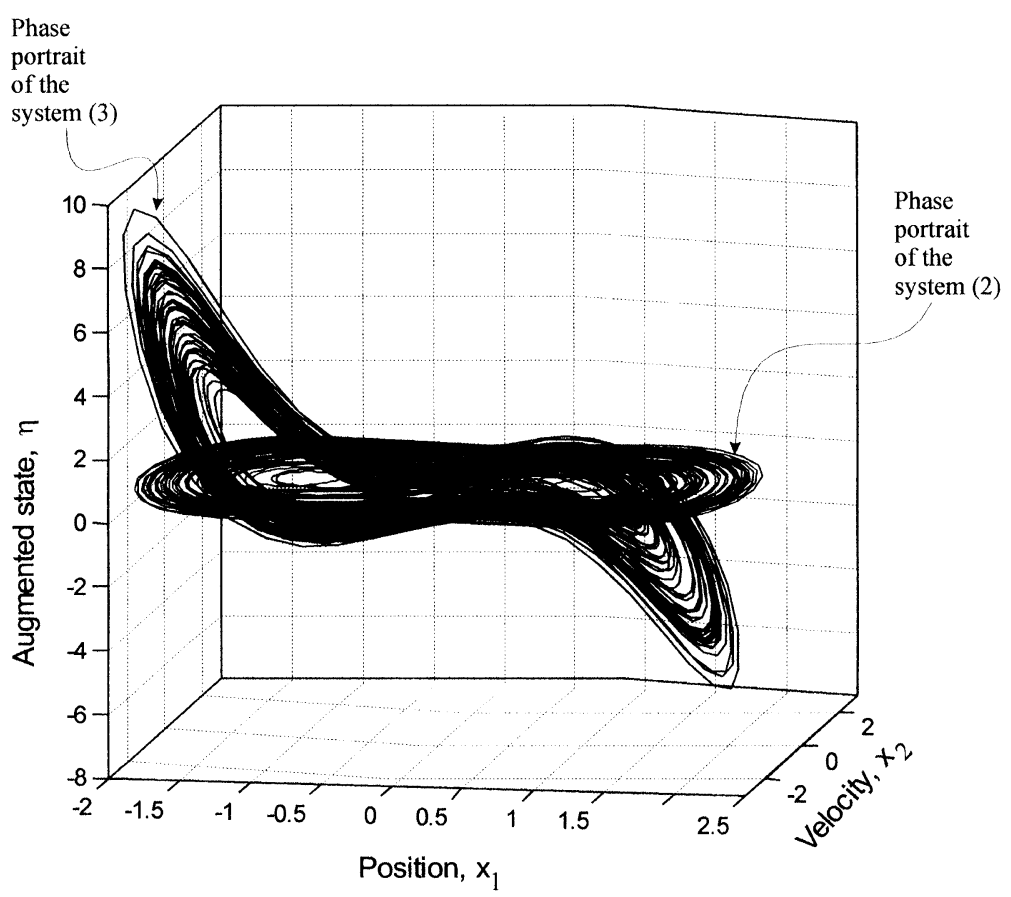

FIGURE 1 Blue line is the space-phase of the Duffing sequation whereas black line represents the trajectories of the Duffing extended space.

feedback requires knowledge about the uncertain terms. Here, the following estimation procedure is proposed: The main idea is to estimate the unmeasured states of the system (3) from measurements of the time series $y=x_{1}$. It is clear that, from Eqs. (3.a) and (3.b), the unmeasured states can be estimated by $x_{2} \approx \hat{x}_{2}=\left(x_{1}\left(t_{i}\right)-x_{1}\left(t_{i-1}\right)\right) / \Delta t$ and $\eta\left(t_{k}\right) \approx \hat{\eta}\left(t_{i}\right)=\left(x_{1}\left(t_{i}\right)+2 x_{1}\left(t_{i-1}\right)-x_{1}\left(t_{i-2}\right)\right) / \Delta t-$ $u\left(t_{i-1}\right)$, where $\Delta t$ denotes the sampling rate, $\left(\hat{x}_{2}, \hat{\eta}\right)$ are estimated values of $\left(x_{2}, \eta\right)$ and $x_{1}\left(t_{i}\right)$ is the value of the measured state at time $t=t_{i}$. In this way the ideal feedback can be modified to get

$u\left(t_{i}\right)=-\hat{\eta}\left(t_{i}\right)+k_{2} x_{1}\left(t_{i}\right)+k_{1}\left(x_{1}\left(t_{i}\right)-x_{1}\left(t_{i-1}\right)\right) / \Delta t$

where $\hat{\eta}\left(t_{i}\right)$ is given by the above estimator. Note that the uncertainties estimator $\hat{\eta}\left(t_{i}\right)=\left(x_{1}\left(t_{i}\right)+\right.$ $\left.2 x_{1}\left(t_{i-1}\right)-x_{1}\left(t_{i-2}\right)\right) / \Delta t+u\left(t_{i-1}\right) \quad$ only requires knowledge about the measured and the last control action. This can be seen as a torque balance. In this sense the estimated value $\hat{\eta}\left(t_{i}\right)$ reconstructs the unknown force acting into (or onto) the system. The feedback (4) and the uncertainties estimator cannot be designed separately. In this way, the unveiling procedure depends on two factors: (a) The feedback parameters should be chosen such that the real part of the roots of the polynomial $P_{2}(s)$ be negative defined and (b) the sample rate $\Delta t$ be arbitrarily small. Indeed, as $\Delta t \rightarrow 0$ as the $u\left(t_{i}\right)$ tends to the ideal feedback, $u$. Of course, if $\Delta t=0$ the feedback (4) cannot be physically realizable. This is, there is a tradeoff between the estimation and the stabilization capability, which can be resumed as: The rate of the uncertainties estimation is limited by stabilization rate and viceversa. The unveiling tradeoff is illustrated in next section.

\section{ILLUSTRATIVE EXAMPLES}

We have selected two interesting systems: The former is the magneto-elastic beam. In this system, 
we illustrate that forces acting onto the system can be reconstructed via the proposed scheme. The latter is a magnetic bearing. In this case, the uncertainties source is into the systems. The unknown function is the force between the magnet and a class of high-temperature superconductor.

\subsection{Unveiling External Perturbing Force}

Let us consider the magneto-elastic beam system. This system was experimentally studied by Moon and Holmes (see Chapter 2 in [2]) and can be modelled by the a driven second-order oscillator (Duffing equation): $\ddot{x}+\delta \dot{x}-x+x^{3}=\tau_{e}(t)+u$, where $\delta$ is a damping coefficient, $u$ denotes the stabilizing command and $\tau_{e}(t)$ is a periodic function, which represents the perturbing external force, e.g., $\tau_{e}(t)=A \cos (\omega t)$. Note that in this case the uncertain force $\alpha(x(t)) \equiv 0$ for all $t$, see Eq. (1.b).

Let us assume that only the perturbing force, $\tau_{e}(t)$, is unknown and unmeasured. In this way, the augmented state is defined by $\eta=\tau_{e}(t)$ and the extended uncertain system becomes

$$
\begin{aligned}
\dot{x}_{1} & =x_{2} \\
\dot{x}_{2} & =\eta-\delta x_{2}+x_{1}-x_{1}^{3}+u \\
\dot{\eta} & =\dot{\tau}_{e}(t)
\end{aligned}
$$

where $\eta$ and its time-derivative are unknown. Thus, the internal model feedback is given by

$$
\begin{aligned}
\hat{\eta}\left(t_{i}\right)= & \frac{x_{1}\left(t_{i}\right)+2 x_{1}\left(t_{i-1}\right)-x_{1}\left(t_{i-2}\right)}{(\Delta t)^{2}} \\
& +\delta\left(\frac{x_{1}\left(t_{i}\right)-x_{1}\left(t_{i-1}\right)}{\Delta t}\right) \\
& +x_{1}\left(t_{i}\right)-x_{1}^{3}\left(t_{i}\right)-u\left(t_{i-1}\right) \\
u\left(t_{i}\right)= & \left(k_{1}+\delta\right)\left(\frac{x_{1}\left(t_{i}\right)-x_{1}\left(t_{i-1}\right)}{\Delta t}\right) \\
& -\hat{\eta}\left(t_{i}\right)-x_{1}\left(t_{i}\right)+x_{1}^{3}\left(t_{i}\right)+k_{2} x_{1}\left(t_{i}\right)
\end{aligned}
$$

Figure 2 shows the performance of the time discrete feedback for several sampling rate, $\Delta t$. The sampling rate were chosen as follows: $\Delta t=0.0005$ (dashed line), $\Delta t=0.01$ (solid line),
$\Delta t=0.1$ (dotted line) and $\Delta t=0.5$ dash-dotted line. The unveiling feedback scheme was activated at $t=50$.

If the uncertain force is unveiled (which means that $\hat{\eta}$ is close to the unknown force), the control action compensates the perturbation onto the system. On contrary, as the $\Delta t$ increases the estimated value of the uncertain term the control action increases and stabilization is lost. For instance, if $\Delta t=0.5$ (see dash-dot line in Fig. 2) then the estimation error is around 1.0, the position, $x_{1}$, cannot be stabilized at origin and the stabilizing command is larger, $u$. This is, the uncertain force can be unveiled if the position error is stabilized at origin and viceversa.

\subsection{Unveiling the Magnetic Force in a Levitation System}

Consider the dynamical system given by

$$
\ddot{x}+\delta \dot{x}+x+\alpha(x, \dot{x}, t)=\tau_{1}(t)+u
$$

where $x$ means the position, $\delta$ is a damping factor, $\tau_{1}(t)=A \cos (\Omega t)$ represents a periodic perturbing force, $\alpha(x, \dot{x}, t)$ is a nonlinear function acting into the system, which represents the force between a magnet supported by the high temperature Type-II superconductor [11].

Let us assume that the internal force, $\alpha(x, \dot{x}, t)$, is uncertain. Besides, consider that external perturbing force, $\tau_{1}(t)$, and damping parameter are exactly known. In addition, suppose that only position, $x_{1}$, is available from measurements. Then, following the above procedure, feedback with uncertainties estimator is obtained

$$
\begin{aligned}
\hat{x}_{2}\left(t_{i}\right)= & \frac{x_{1}\left(t_{i}\right)-x_{1}\left(t_{i-1}\right)}{\Delta t} \\
\hat{\eta}\left(t_{i}\right)= & \frac{x_{1}\left(t_{i}\right)+2 x_{1}\left(t_{i-1}\right)-x_{1}\left(t_{i-2}\right)}{\Delta t^{2}} \\
& +\delta \hat{x}_{2}\left(t_{i}\right)+x_{1}\left(t_{i}\right)-\tau_{1}\left(t_{i}\right)-u\left(t_{i-1}\right) \\
u\left(t_{i}\right)= & -\hat{\eta}\left(t_{i}\right)+\left(\delta-k_{2}\right) \hat{x}_{2}\left(t_{i}\right) \\
& +\left(k_{1}-1\right) x_{1}\left(t_{i}\right)-\tau_{1}\left(t_{i}\right)
\end{aligned}
$$



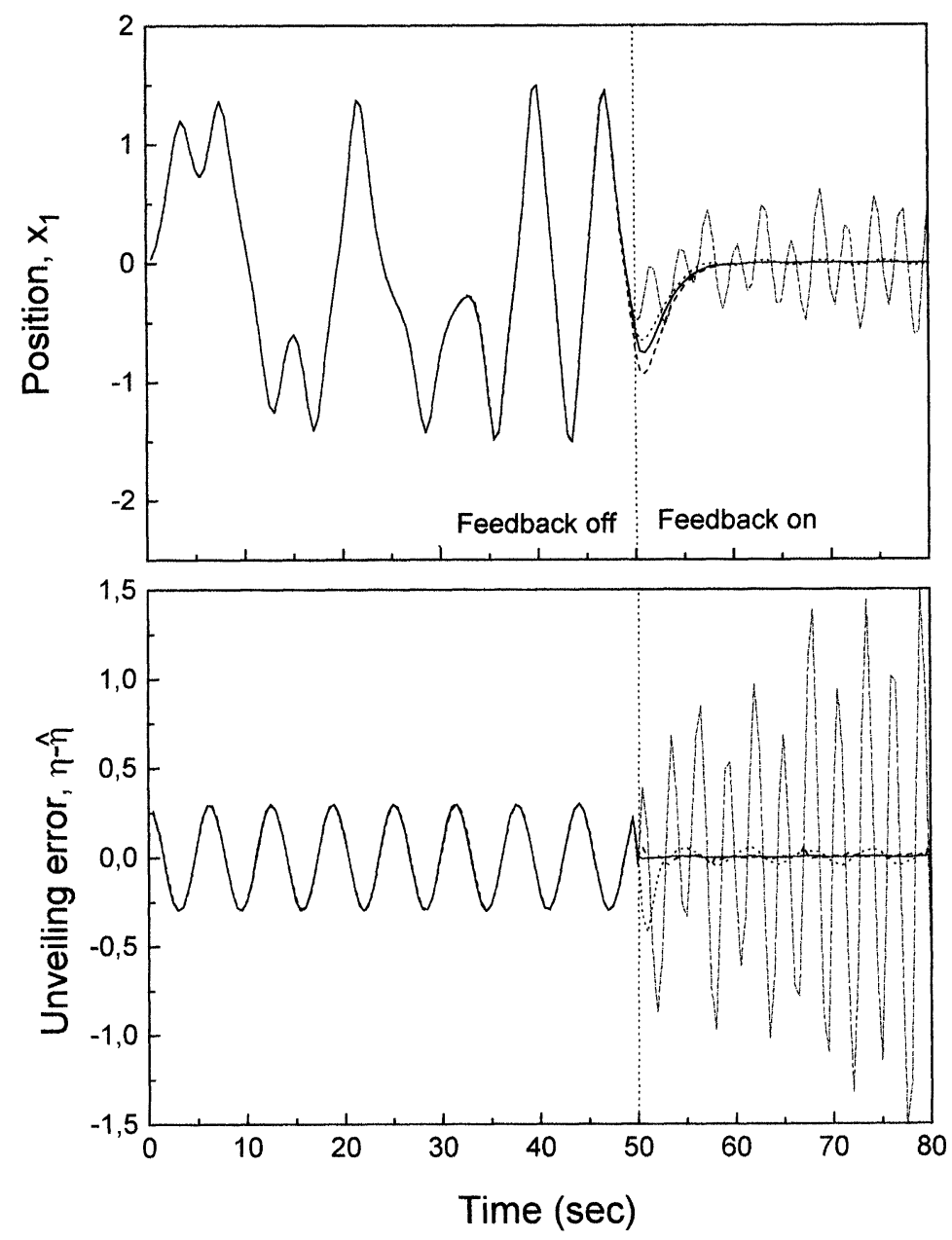

FIGURE 2 Performance of the discrete-time unveiling scheme. The force acting onto the Duffing equation is unveiled.

where the feedback constants are $k_{1}=1.0, k_{2}=2.0$, which implies that the roots of the polynomial $P_{2}(s)$ are located at -1.0 .

In principle, the feedback (6) can be experimentally implemented in a magnetic bearing device. However, in seek of clarity, we have performed numerical simulations of the magnetic levitation process. Although $\alpha(x, \dot{x}, t)$ is not exactly known, it has been reported [11] that the characteristics of the system can depend on the hypo-elasticity function whose dynamics is given by $\dot{\alpha}(x, \dot{x})=$ $\mu_{1}[\alpha(x, \dot{x}, t)-\phi(x, \dot{x})]$, where $\mu_{1}$ is a parameter and the nonlinear function is given by $\phi(x, \dot{x})=\phi_{1}(x)\left[1+\phi_{2}(\dot{x})\right]$. Besides, the nonlinear functions can be approximated by the force-displacement relation, $\phi_{1}(x)=\mu_{2} \exp (-x)$, and

$$
\phi_{2}(\dot{x})=\left\{\begin{array}{cc}
-\mu_{3}-\dot{x}, & \zeta \leq \dot{x} \\
\left(-\dot{x}\left(\mu_{3}+\mu_{4}\right) / 2 \zeta\right), & -\zeta \leq \dot{x}<\zeta \\
\mu_{4}, & \dot{x}<-\zeta
\end{array}\right.
$$

where $\zeta=0.005, \mu_{1}=0.1, \mu_{2}=0.3, \mu_{3}=\mu_{4}=1.0$.

Figure 3 shows the performance of the uncertain force for several sampling rates, $\Delta t$. In this case, if sampling rate $\Delta t=0.5$ the closed-loop system is 

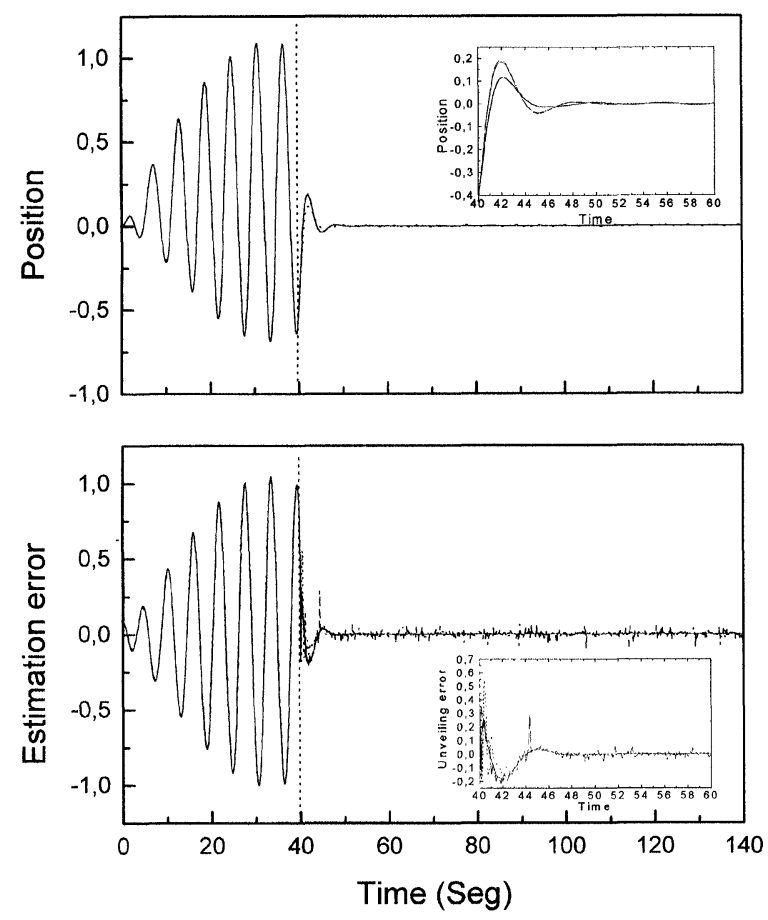

FIGURE 3 Performance of the discrete-time unveiling scheme. The force acting into the magnetic-bearing equation is unveiled.

unstable; hence the sampling rate were chosen as follows: $\Delta t=0.0005$ (dashed line), $\Delta t=0.01$ (solid line) and $\Delta t=0.1$ (dotted line). The unveiling feedback was activated for $t \geq 100$. Note that as the sampling rate decreases the stabilizing command increases, in consequence the unveiling error increases. This is, if $\Delta t \rightarrow 0$ the stabilizing command approach is not physically realizable. Hence, the hidden forces cannot be unveiled.

\section{CONCLUDING REMARKS}

A procedure to construct an unveiling scheme was proposed in this letter. The unveiling is a discretetime feedback scheme and comprises an estimation procedure and a stabilizing command. The procedure departs defining an augmented state to get the construction of an extended system, which is an uncertain nonlinear system. After that, the augmented state is estimated via backward finite differences. In this way, the resulting scheme only requires measurements of one available state at time $t_{i}, t_{i-1}$ and $t_{i-2}$ and the knowledge about last stabilizing command, $u_{i-1}$. Two illustrative examples were presented. In first one an uncertain force acting onto the system is unveiled whereas in the second one an unknown force acting into the system is unveiled.

In principle, the unveiling scheme allows to get an estimated value of any uncertain force from on-line measurements. Hence, experimental implementation can be expected. Previously, the discrete-time unveiling scheme should be designed. Then, the following configuration for experimental unveiling can be proposed: The measurements from the experimental apparatus are entered to any computing machine, where the model and the discrete-time unveiling are programmed. The goal is to synchronize the experimental system and the programmed model via the discrete-time scheme. If synchronous behavior is attained, the estimated 
value of the uncertain force is close to the actual value. Experimental results will be reported in short time.

\section{References}

[1] Haken, H., Synergetics an introduction, 3rd Edition, Springer-Verlag, Berlin, 1983.

[2] Gukenheimer, J. and Holmes, P., Nonlinear Oscillations Dynamical Systems and Bifurcations of Vector Fields, 3rd Edition, Springer-Verlag, New-York, 1983.

[3] Andronov, A. A., Vitt, A. A. and Khaikin, S. E., Theory of Oscillators, Dover, New-York, 1987.
[4] Nijmeijer, H. and Mareels, I. M. Y. (1997). Trans on IEEE Circ. and Syst. I., 44, 882-890.

[5] Letellier, C., Maquet, J., Le Sceller, L., Gouesbet, G. and Aguirre, L. A. (1998). J. Phys. A. Math. Gen., 31, $7913-7927$.

[6] Breeeden, J. L. and Hübler, A. (1990). Phys. Rev. A, 42, $5817-5826$

[7] Takens, F., In: Dynamical Systems and Turbulence, Vol. 898 of the Lecture notes in Mathematics, Eds. Rand, D. and Young, L. S., Springer-Verlag, Berlin, 1981.

[8] Alvarez-Ramírez, J., Femat, R. and González, J. (1996). Phys. Letts A, 211, 41-46.

[9] Goodwin, G. C. and Seron, M. M. (1997). Trans. on Aut. Control, 42, 1240-1251.

[10] Femat, R. (1998). Physica D, 111, 347-355.

[11] Hikihara, T. and Moon, J. R. (1994). Phys. Letts A, 191, 249. 


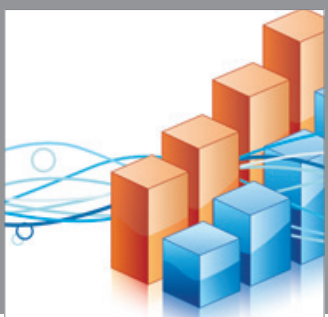

Advances in

Operations Research

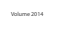

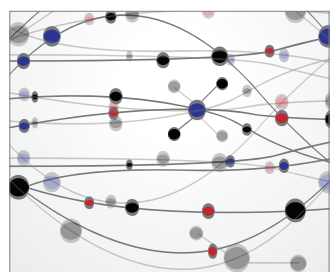

\section{The Scientific} World Journal
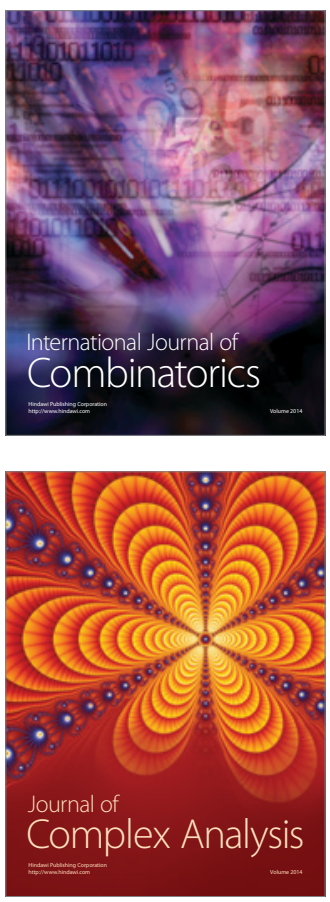

International Journal of

Mathematics and

Mathematical

Sciences
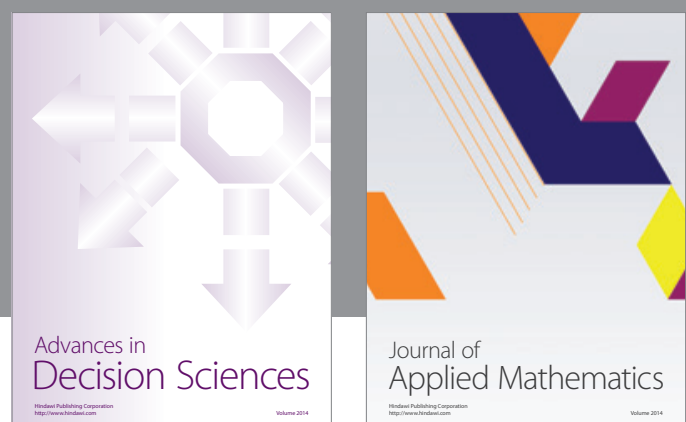

Journal of

Applied Mathematics
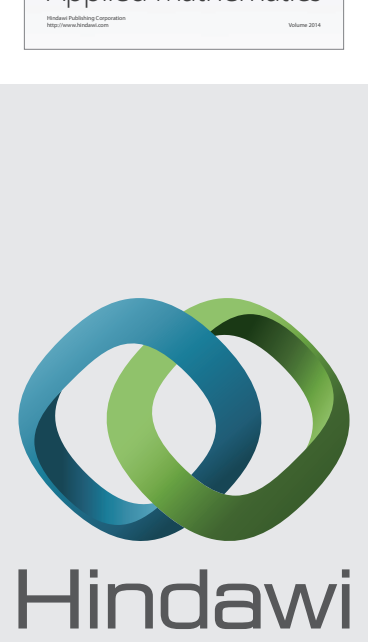

Submit your manuscripts at http://www.hindawi.com
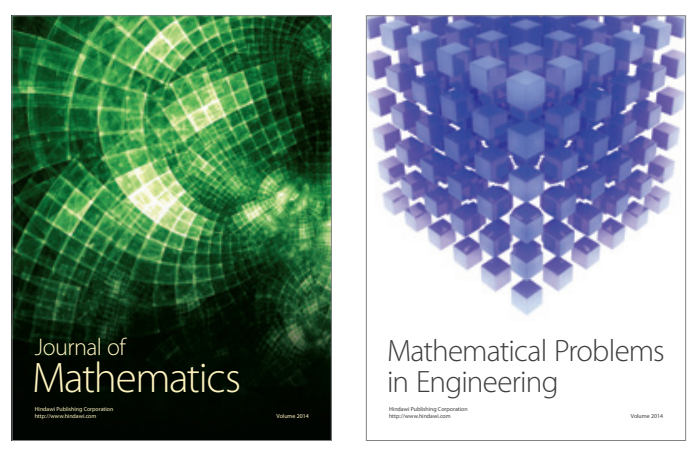

Mathematical Problems in Engineering
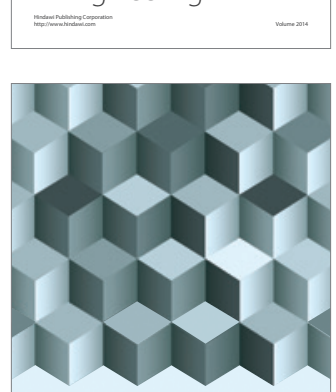

Journal of

Function Spaces
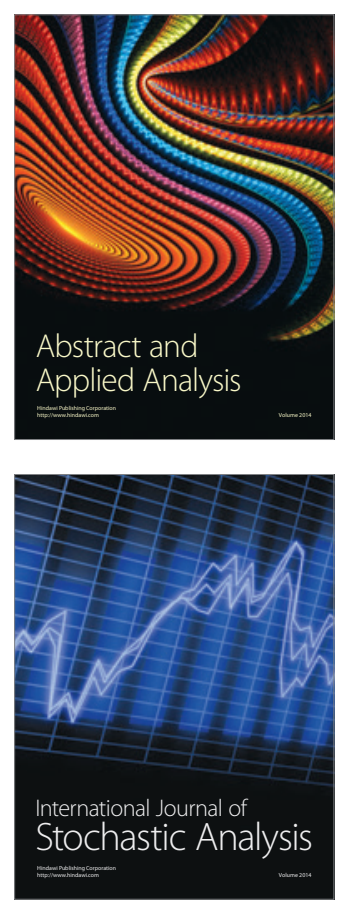

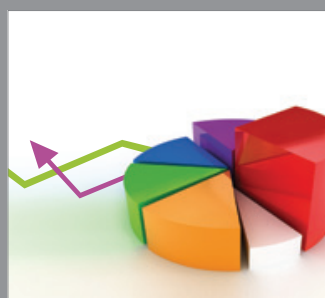

ournal of

Probability and Statistics

Promensencen
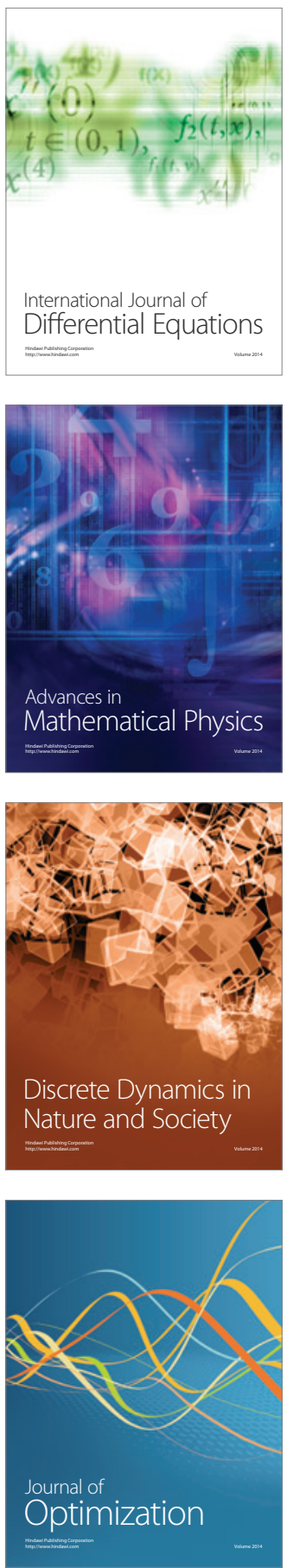